\title{
Correspondence
}

\section{Relative hepatic weight using body weight may be not accurate}

$\mathrm{W}$ e read the paper entitled "A high-fat highenergy diet influences hepatic CYP3A expression and activity in low-birth-weight developing female rats" published in the recent issue of the Journal. ${ }^{[1]}$ The authors investigated the effects of a high-fat, high-energy diet (HFHE diet) on the hepatic expression of CYP3A in low-birth-weight developing female rats. And they concluded that an HFHE diet can result in alterations of CYP3A expression in a developmental low birth-weight rat model.

I have some concerns for Ni et al's study. Firstly, why the study only performed in female rats? The authors did not explain the reason they only select the female rats for this study. How about the effects of HFHE on male rats? It is very important for the authors to discuss this issue in the paper. In my view, the results obtained from only male rats may have some bias. Sugatani et $\mathrm{al}^{[2]}$ found that $\mathrm{mRNA}$ levels of CYP3A1/2 were markedly decreased in HF dietfed and fluvastatin-treated rats. Yoshinari et $\mathrm{al}^{[3]}$ showed that one-week administration of a high-fat diet in rats also reduced hepatic CYP3As. Secondly, the authors described the hepatic index as liver weight per unit of body weight, however the correct form for the relative hepatic weight is using the tibial length. Yin et $\mathrm{al}^{[4]}$ performed a study on using tibial length to quantify cardiac hypertrophy. Under conditions in which body weight changes, relative hepatic weight can be more accurately quantified by relating hepatic weight to tibial length than to body weight; and this approach can be used for assessing relative sizes of other organs as well. ${ }^{[4]} \mathrm{Ni}$ et al ${ }^{[1]}$ used body weight to quantify hepatic weight in their study. However, the diet used in the study may have affected body weight a lot. Therefore, the relative hepatic weight in their study may be not so accurate.

\author{
Xiu-Juan Yang \\ Department of Pediatrics, \\ The First Affiliated Hospital of Zhejiang Chinese Medical University, \\ Hangzhou, China \\ Email: ateate221155@126.com
}

\section{References}

1 Ni SQ, Lou Y, Wang XM, Shen Z, Wang J, Zhao ZY, et al. A high-fat high-energy diet influences hepatic CYP3A expression and activity in low-birth-weight developing female rats. World J Pediatr 2016;12:489-497.

2 Sugatani J, Sadamitsu S, Kurosawa M, Ikushiro S, Sakaki T, Ikari A, et al. Nutritional status affects fluvastatin-induced hepatotoxicity and myopathy in rats. Drug Metab Dispos 2010;38:1655-1664.

3 Yoshinari K, Takagi S, Yoshimasa T, Sugatani J, Miwa M. Hepatic CYP3A expression is attenuated in obese mice fed a high-fat diet. Pharm Res 2006;23:1188-1200.

4 Yin FC, Spurgeon HA, Rakusan K, Weisfeldt ML, Lakatta EG. Use of tibial length to quantify cardiac hypertrophy: application in the aging rat. Am J Physiol 1982;243:H941-H947.

doi: 10.1007/s12519-017-0022-9

$\mathrm{W}$ e wrote the paper entitled "A high-fat highenergy diet influences hepatic CYP3A expression and activity in low-birth-weight developing female rats". ${ }^{[1]}$ As sex has influence on the expression of animal's CYP3A, the samples of experimental female and male pups were collected the same way but separately. ${ }^{[1]}$ If we included the sex variants in this paper, there would be too many groups (14 groups) and variants (ontogeny, diet, sex), and it was very difficult to explain the results. As a result, in this paper, we only reported the results of female samples.

Sugatani et $\mathrm{al}^{[2]}$ used male, 6-weeks age Wistar rats to study whether nutritional status affects adverse effects of fluvastatin. Rats were fed with a standard diet (SD diet) [12.9\% lipid, $60.4 \%$ carbohydrate, and $26.7 \%$ protein (kilojoules)] or high-fat and high-sucrose diet (HF diet) $[23.9 \%$ lipid, $56.8 \%$ carbohydrate, and $19.3 \%$ protein (kilojoules)] for one week, administered fluvastatin with the diet for another 4,8 , or 14 days. This study found that the mRNA levels of CYP3A1/2 were markedly decreased in HF diet; and Yoshinari et al's study suggested that the constitutive expression of CYP3A in 5-week-old mice that were given with a high-fat diet (36\% fat, $22 \%$ protein, and $35 \%$ carbohydrate or $32 \%$ fat, $26 \%$ protein, and $29 \%$ carbohydrate) for 35-40 days drastically reduced both mRNA and protein levels. ${ }^{[3]}$ One-week administration of the same high-fat diet to 9-week-old mice or rats also reduced hepatic CYP3A. ${ }^{[3]}$ In our study, the highfat and high energy diet (HFHE diet) included 16.4\% fat, $21.3 \%$ protein, and $53.7 \%$ carbohydrate, and the quality of fat and other ingredients differed from the above-mentioned two researches; the animal species 